Published in final edited form as:

J Acquir Immune Defic Syndr. 2010 February ; 53(Suppl 1): S10-S14. doi:10.1097/QAI.

0b013e3181c7d363.

\title{
The prevalence of transmitted antiretroviral drug resistance in treatment naïve HIV-infected individuals in China
}

\author{
Lingjie Liao, PhD*, Hui Xing, MD*, Hong Shang, MD, PhD ${ }^{\dagger}$, Jingyun Li, PhD ${ }^{\ddagger}$, Ping Zhong, \\ PhD $\$$, Hua Cheng, PhD*, Xinping Li, MD*, and Yiming Shao, MD, PhD* \\ "State Key Laboratory for Infection Diseases Prevention and Control, National Center for AIDS/ \\ STD Control and Prevention (NCAIDS), Chinese Center for Disease Control and Prevention \\ (China CDC), Beijing, China \\ †Key Laboratory of Immunology of AIDS, Ministry of Health, the First Affiliated Hospital, China \\ Medical University, Shenyang, Liaoning province, China \\ ¥AIDS Research Department, Institute of Microbiology and Epidemiology, Academy of Military \\ Medical Science, Beijing, China \\ $\S$ Department of AIDS and STD, Shanghai Municipal Center for Disease Control and Prevention, \\ Shanghai, China
}

\begin{abstract}
Background-Transmission of HIV drug resistance (TDR) gives rise to reduced efficacy of initial antiretroviral treatment, and has become a public health concern.

Methods-A nationwide survey on TDR was conducted in antiretroviral therapy naïve HIV-1 infected individuals from September 2004 to October 2005 in China. Drug resistance genotyping was performed on subjects' plasma samples. Drug resistance mutations were determined and scored by Stanford HIV Drug Resistance algorithm.
\end{abstract}

Results-Sequences were obtained from 676 individuals, of which $61.2 \%$ were former plasma and blood donors, $17.3 \%$ were infected sexually, and $17.2 \%$ were intravenous drug users. Subtype B' HIV-1 strains were found in 73.5\%, CRF01_AE in 13.9\%, CRF07_BC in 6.2\%, CRF08_BC in $2.7 \%$, subtype $\mathrm{C}$ in $1.04 \%$, subtype $\mathrm{B}$ in $0.9 \%$, CRF02_AG in $0.4 \%$ and $\mathrm{B}$ '/C intersubtype recombinant strains in $1.3 \%$ of the subjects. Twenty-six (3.8\%) were found to harbor drug resistance strains. The rates of resistance to PIs, NRTIs and NNRTIs were $0.4 \%, 1.6 \%$ and $2.1 \%$, respectively. Though there was no significant difference in TDR rates between 2004 and 2005 ( $2.9 \%$ vs. $4.4 \%)$, an increased trend was observed in the rate of high-level drug resistance $(0.8 \%$ in 2004 vs. $3.0 \%$ in $2005, \mathrm{P}=0.0634$ ).

Conclusions-The rate of TDR was relatively low in China, as compared with those in developed countries. Surveys among recently HIV-infected subjects should be preformed continually to ensure the success of the scale-up antiretroviral treatment.

\section{Keywords}

treatment naïve; transmitted HIV-1 drug resistance; prevalence

\footnotetext{
Correspondence author: Yiming Shao, MD, PhD, State Key Laboratory for Infection Disease Prevention and Control, National Center for AIDS/STD Control and Prevention (NCAIDS), Chinese Center for Disease Control and Prevention (China CDC), Beijing 100050, People's Republic of China (yshao@bbn.cn).

Presented in part at the 4th IAS Conference on HIV Pathogenesis, Treatment and Prevention, Sydney, Australia, July 22-25, 2007 [abstract TUPEB051].
} 


\section{INTRODUCTION}

Since the first human immunodeficiency virus (HIV) case was identified in 1985 in China, the HIV/acquired immunodeficiency syndrome (AIDS) epidemic kept rising during the subsequent twenty years. By the end of 2005, it was estimated that 650,000 individuals living with HIV/AIDS in China, of whom 75,000 were AIDS patients. ${ }^{1}$ Intravenous drug use $(44.3 \%)$, sexual contact (43.6\%), and unsafe blood and plasma collection (10.7\%) were main transmission routes which contributed to most of the 141,000 HIV cases reported.

Furthermore, the distribution of HIV/AIDS cases was not geographically even in China: five out of 31 provinces and autonomous regions had reported over 10,000 cases, while three other provinces had less than 100 reported cases.

To improve the life of HIV/AIDS patients and reduce the HIV/AIDS-related morbidity and mortality, the Chinese government put forward a "four free one care" policy in $2003 .^{2}$ About 5,000 patients received a national free antiretroviral therapy (ART) program in its first year of inception. Since then, the number of patients enrolled in the program has increased rapidly. At the end of 2005, more than 22,000 patients were ever involved in the free ART program, of which about 19,200 were still on ART. ${ }^{3}$ Most of them were HIV-infected former blood and plasma donors (FPDs) living in rural areas of central China. Resources for antiretroviral drugs are limited in China. There is a concern that the prevalence of antiretroviral drug resistance would compromise the effect of current regimens and give rise to treatment failure.

With the scale-up of a free ART program, a nationwide survey was conducted to study the genetic characteristic and antiretroviral drug resistance of HIV type 1 (HIV-1) strains in treatment naïve HIV-infected individuals from 2004 to 2005.

\section{METHODS}

\section{Study population}

Treatment naïve HIV-1-infected individuals were randomly enrolled in provinces and autonomous regions where free antiretroviral drugs were available at the time the survey was conducted. The duration of sample collection was from September 2004 to October 2005. In 2004, treatment naïve infected individuals in 14 provinces were included. With the rapid scale-up of the national free ART program, the geographic range of the study was expanded to 28 provinces and autonomous regions in the subsequent year. Subjects who were below 18 years old were excluded from the study. All the subjects gave written informed consent for participation in this study. The survey was reviewed and approved by the institutional review boards and scientific ethical committees at the Chinese Ministry of Health.

\section{Laboratory tests}

Subjects' whole blood samples were collected and processed in the provincial CDCs' labs. CD4 cell count was measured within 24 hours after sampling. Meanwhile, plasma was separated by centrifugation and instantly stored at $-80^{\circ} \mathrm{C}$. The plasma samples were then sent in dry ice to the four core laboratories of the Chinese HIV Drug Resistance Surveillance Network (CN-HIVResNet). Plasma HIV RNA was quantified with real-time NASBA (NucliSense Easy Q, bioMerieur, France) or COBAS (Roche Applied Science, Germany) according to the manufacturer recommendations. For samples with viral loads equal to or greater than 1000 copies /mL, HIVDR genotyping was carried out by an in-house PCR protocol as previously described. ${ }^{4} \mathrm{HIV}-1 \mathrm{pol}$ gene (protease 1-99 amino acids and part of reverse transcriptase 1-250 amino acids) was amplified, purified and bidirectly sequenced in an ABI3100 sequencer (Applied Biosystems, Foster City, CA). For analysis of HIV-1 drug 
resistance mutation, each sample sequence was compared with the subtype B consensus sequence in the Stanford HIV Drug Resistance Database (http://hivdb.stanford.edu) and was interpreted by HIVdb program. For HIV-1 subtyping, the edited sequences were aligned against reference sequences available at the Los Alamos database (http://hivweb.lanl.gov). Phylogenetic trees were constructed by the neighbor-joining method with 1000 bootstrap replicates using Mega 4.0. Bootscaning analyses were performed on samples possibly having unidentified intersubtype recombinant strains by using Simplot 3.5.1.

\section{Statistical analysis}

The significant differences in categorical variables were analyzed using chi-square test or Fisher's exact tests. Statistical significance was defined as a $P$ value $<0.05$. All the statistical analyses were performed with SAS 9.1 software (SAS Institute, Cary, NC, USA).

\section{RESULTS}

\section{Subjects included}

One thousand, one hundred and ninety-four ART naïve HIV-1-infected subjects were enrolled (442 in 2004 and 752 in 2005). Two hundred and twenty-seven individuals who had a plasma viral load less than $1000 \mathrm{copies} / \mathrm{ml}$ were excluded from the study, as the possibility of such subjects to be sources of HIV infection would be less than those with a higher viral load. In addition, the genotypic results from samples with a viral load lower than 1000 copies $/ \mathrm{ml}$ were not reliable using routine methods. Of the remaining 967 samples, 676 (69.9\%) were successfully sequenced. Among these subjects, 460 (68.1\%) were male. Median age was 38 years (range: 18-72). Four hundred and fourteen (61.2\%) were former plasma donors (FPDs), 117 (17.3\%) acquired HIV infection through sexual contact, 116 (17.2\%) were intravenous drug users, $27(4.0 \%)$ were infected through blood transfusion, and the transmission routes of the remaining two subjects were unknown. More than half of the subjects $(351 / 676,51.9 \%)$ had a primary school education or less. Four hundred and fifty-one (66.7\%) were farmers living in rural areas. Median CD4 cells count and viral load at sampling were 280 cells/mm3 (range: 23-723), and $4.59 \mathrm{log}$ copies/ml (range: 3.01-6.80), respectively.

\section{HIV-1 subtypes, circulating recombinant forms, or intersubtype recombinant strains}

Phylogenetic trees base on the whole protease (PR, 1-99aa) and part of reverse transcriptase (RT, 1-250aa) gene revealed that a majority of the HIV-1 isolates $(497,73.5 \%)$ studied belonged to subtype Thai-B ( B'), the second most prevalent strain was CRF01_AE (94, $13.9 \%)$, followed by CRF07_BC (42, 6.2\%), CRF08_BC (18, 2.7\%), subtype C (7, 1.04\%), subtype B $(6,0.9 \%)$ and CRF02_AG $(3,0.4 \%)$. Bootscaning analyses were performed on sequences from nine samples, which did not cluster with any present known reference sequences. The results revealed the presence of B'/C intersubtype recombinant strains with different mosaic structures from those of CRF07_BC and CRF08_BC (Fig. 1). The distribution of HIV-1 strains was uneven among different risk groups $(P<0.0001)$. Subtype B' HIV-1 strains were more frequently found in FPDs $(398,80.1 \%)$ than in other HIV-1infected populations, CRF07_BC strains were more likely to be found among intravenous drug users (IDUs) $(27,64.3 \%)$, while most CRF01_AE strains were detected in two groups: IDUs $(46,39.7 \%)$ and sexually infected individuals $(41,35.0 \%)$.

\section{Antiretroviral drug resistance-associated mutations}

Twenty-six (3.8\%) out of 676 isolates were identified to be resistant to one or more antiretroviral drugs (Table 1). Primary drug resistance mutations to PIs were found in three $(0.4 \%)$ samples, which had one single mutation each. M46I and N88D, which confer 
intermediate resistance to NFV, were respectively detected in one subject (CNSHD0050) from Shanghai and one person (CNHND0066) from Hunan province. A V82A mutation conferring resistance to ATV, IDV, LPV, and NFV was found in one subject (CNGXD0011) from Guangxi province.

Mutations associated with resistance to reverse transcriptase inhibitors were found in 23 subjects. Twenty one of them were resistant to either NRTIs $(n=9)$ or NNRTIs $(n=12)$; two were resistant to both NRTIs and NNRTIs. The most frequent NRTI-related mutation was M184V/I, conferring high resistance to 3TC and FTC, and was found in four (4/23, $17.4 \%$ ) samples. At least one thymidine analogue mutation (TAM), which was associated with intermediate or low level resistance to AZT or other NRTIs, was found in five samples. The most frequent NNRTI-related mutation was K103N, which was found in nine $(9 / 23$, $39.1 \%)$ samples.

Comparison of the HIVDR rate in three major risk groups revealed that there was no significant difference among FPDs (13/414, 3.1\%), sexually infected individuals (5/117, $4.3 \%)$ and IDUs $(4 / 116,3.5 \%)$. No significance difference on transmission of HIV drug resistance (TDR) rate was found between female (7/216, 3.2\%) and male (19/460, 4.1\%) subjects, those with primary education or below (3.7\%), and those with a higher education $(4.0 \%)$, or between non-farmers $(5.3 \%)$ and farmers (3.1\%). The rate of TDR in 2004 and 2005 was $2.9 \%$ (7/244) and 4.4\% (19/432), respectively, with no significant difference between the two years $(P=0.3207)$. The rate of high-level resistance to one or more antiretroviral drugs was also not significant $(\mathrm{p}=0.0634)$, although there was an increase in 2005 (3.0\%, 13/432), compared with 2004 (0.8\%, 2/244).

\section{DISCUSSION}

Our study was the first nationwide survey on TDR in ART naïve individuals and revealed the existence of transmitted HIV drug resistance in China. Evidence of TDR was found in $3.8 \%$ of the subjects studied. This result was similar to results from a previous survey, which found $4.4 \%$ of HIV drug resistance out of 91 ART naïve HIV/AIDS patients in Liaoning, a province in northeastern China. ${ }^{5}$ This prevalence was comparable to that of international studies which showed 2.0\% TDR during 2003 and 2006 in Thailand, ${ }^{6} 4.3 \%$ from 1999 to 2005 in South Korea, ${ }^{7}$ and $4.3 \%$ in 2002 in the Democratic Republic of Congo. ${ }^{8}$ However, the prevalence of TDR in our study was lower than those in North America and Western Europe where various TDR rates were reported to be from $9.1 \%{ }^{9}$ to $24.1 \%{ }^{10}$ The result was not surprising, as China, like other countries in Asia and Africa, has had a shorter period of access to antiretroviral therapy than in developed countries.

In this study, the largest proportion of the HIV-1 strains was subtype B' strains, followed by CRF01_AE, CRF07_BC, CRF08_BC and other subtypes, circulation recombinant forms or intersubtype recombinant forms. Though the total proportion of four predominant HIV-1 strains (subtype B', CRF01_AE, CRF07_BC and CRF08_BC) and that of CRF01_AE strains alone were similar to what we previously reported on the second nationwide molecular epidemiology survey in 2001 and 2002, ${ }^{11}$ the proportion of specific HIV-1 strains was different: CRF_BC strains, including CFR07_BC and CRF08_BC, accounted for more than half of the HIV-1 infections, subtype B' only contributed to about one third of the infections in that molecular epidemiology survey. This discrepancy may be due to a sampling bias in favor of regions where access to antiretroviral therapy had been already established before the study began. Additionally, the shift of risk groups of HIV-infection that had occurred in recent years may produce an influence in the distribution of HIV-1 strains to some extent. ${ }^{12}$ For this study, subtype B' strains also contributed to a substantial proportion of HIV-1 infections through sexual route and intravenous drug use. 
Half (13/26) of the HIV drug resistance was found in individuals who were infected by unsafe commercial blood and plasma donation. It was believed that most FPDs acquired HIV infection in the mid-1990s, although a large number of HIV infections were confirmed during the intensive screening of FPDs in 2004. Antiretroviral drugs was not introduced in China during the 1990s. It was therefore impossible that these patients were infected with drug resistance viruses at that time. To avoid including subjects who had a history of ART, the ART status of all subjects with detected drug resistance were re-investigated by phone or interviewing. About 30 persons with drug resistance were excluded from the study because of having taken antiretroviral drugs. The existence of HIV drug resistance in FPDs in this study include several subjects who were infected through intravenous drug use or sexual contact and were reluctant to confess to their mode of transmission due to social stigma. ${ }^{13,14}$ A portion of FPDs with drug resistance had actually taken antiretroviral drugs at least once, but they were not fully able to understand the questions about their treatment status, or they didn't think they had been treated because they only took antiretroviral drugs once or twice. The latter situation would lead to overestimate the prevalence of TDR.

The results for this study was not generalizable to overall HIV infections for the following reasons. Firstly, a large proportion of the subjects were not infected recently. As drug resistance strains become minor quasispecies in the plasma after a period of infection, the regular population-based sequencing method will fail to detect them. ${ }^{15}$ Secondly, the selection of the studied population could skew the estimate of the TDR rate in HIV-1 infections. Thirdly, a relative low amplification rate of samples with viral loads equal to or greater than 1000 copies/ml may impose an impact on the evaluation of TDR rate. Analysis of the distribution among risk groups revealed that the proportion of the unamplified samples from IDUs and subjects who were infected sexually was higher than those successfully amplified $(P=0.0008)$. However, our data had shown that there was no significant difference on the TDR rate in these three populations.

In this survey, we demonstrated the presence of TDR among treatment naïve individuals. More than half of the sample with HIV drug resistance showed resistance to NNRTI drugs. Though the TDR rate was relatively low comparing to those in developed countries, comprehensive measures are needed to prevent the spread of HIV drug resistance. More studies should be performed to investigate the rate of TDR among recent infections and provide implications to first-line therapy.

\section{Acknowledgments}

We appreciate the contribution of all subjects included in this study. We are greatly grateful to the staff from provincial and county CDCs for participating in sample collection and investigation. We thank Dr. Adrian Liau for his academic editing of the manuscript.

Supported by funds from the Ministry of Health of the People's Republic of China, the Mega-projects of Science Research for the 11th five-year plan (2008ZX10001-004), the Global Fund to Fight AIDS, Tuberculosis and Malaria, and the China ICOHRTA with Grant \#U2R TW 006918, funded by the US National Institutes of Health, the Fogarty International Center, the National Institute of Drug Abuse and the National Institute of Mental Health.

\section{REFERENCES}

1. Ministry of Public Health, People's Republic of China, Joint United Nations Programme on HIV/ AIDS, World Health Organization, 2005 Update on the HIV/AIDS Epidemic and Response in China. 2006 Jan 24.

2. Zhang FJ, Pan J, Yu L, Wen Y, Zhao Y. Current progress of China's free ART program. Cell Res. 2005; 15:877-882. [PubMed: 16354563]

3. Zhang F, Haberer JE, Wang Y, et al. The Chinese free antiretroviral treatment program: challenges and responses. AIDS. 2007; 21(Suppl 8):S143-S148. [PubMed: 18172383] 
4. Zhong P, Pan Q, Ning Z, et al. Genetic diversity and drug resistance of human immunodeficiency virus type 1 (HIV-1) strains circulating in Shanghai. AIDS Res Hum Retroviruses. 2007; 23:847856. [PubMed: 17678466]

5. Han X, Zhang M, Dai D, et al. Genotypic resistance mutations to antiretroviral drugs in treatmentnaive HIV/AIDS patients living in Liaoning Province, China: baseline prevalence and subtypespecific difference. AIDS Res Hum Retroviruses. 2007; 23:357-364. [PubMed: 17411368]

6. Apisarnthanarak A, Jirayasethpong T, Sa-nguansilp C, et al. Antiretroviral drug resistance among antiretroviral-naive persons with recent HIV infection in Thailand. HIV Med. 2008; 9:322-325. [PubMed: 18400079]

7. Choi JY, Kim EJ, Park YK, Lee JS, Kim SS. National survey for drug-resistant variants in newly diagnosed antiretroviral drug-naive patients with HIV/AIDS in South Korea, 1999-2005. J Acquir Immune Defic Syndr. 2008; 49:237-242. [PubMed: 18845957]

8. Vidal N, Mulanga C, Bazepeo SE, et al. HIV type 1 pol gene diversity and antiretroviral drug resistance mutations in the Democratic Republic of Congo (DRC). AIDS Res Hum Retroviruses. 2006; 22:202-206. [PubMed: 16478404]

9. SPREAD programme. Transmission of drug-resistant HIV-1 in Europe remains limited to single classes. AIDS. 2008; 22:625-635. [PubMed: 18317004]

10. Shet, A.; Rowe, L.; Hogan, C., et al. Transmission of drug resistant HIV-1 among subjects with acute and early HIV-1 infection in 2003 to 2004 [abstract 289]. Presented at, 12th Conference on Retroviruses and Opportunistic Infections; Boston. 2005.

11. Research progress: Nationwide HIV molecular epidemiology survey and its database setting. Ministry of Science and Technology, China. 2004 May 30. Available at: http://shgy.jhgl.org/ shownews.asp? newsid=893.

12. State council AIDS working committee office, UN theme group on AIDS in China: A joint assessment of HIV/AIDS prevention, treatment and care in China, (2007). 2007 Dec 1.

13. Tucker JD, Ren X. Sex worker incarceration in the People's Republic of China. Sex Transm Infect. 2008; 84:34-35. discussion 6. [PubMed: 18212187]

14. Yin L, Zhang Y, Qian HZ, et al. Willingness of Chinese injection drug users to participate in HIV vaccine trials. Vaccine. 2008; 26:762-768. [PubMed: 18191881]

15. Booth CL, Geretti AM. Prevalence and determinants of transmitted antiretroviral drug resistance in HIV-1 infection. J Antimicrob Chemother. 2007; 59:1047-1056. [PubMed: 17449483] 


\section{CNYND0019}

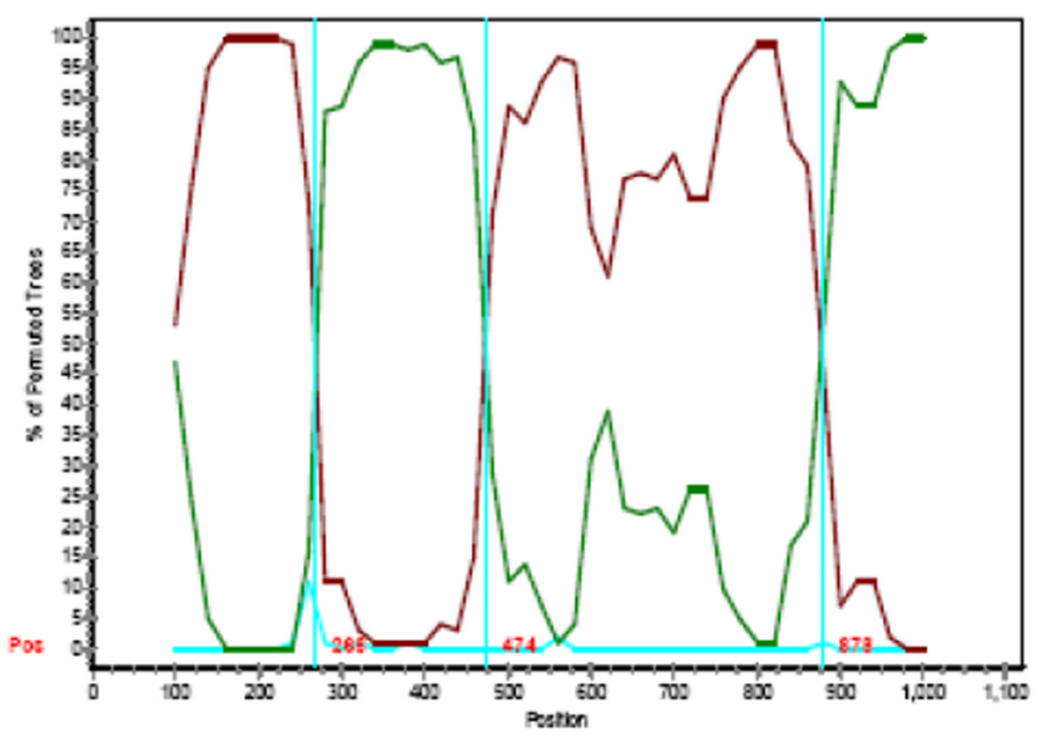

\section{CNYND0041}

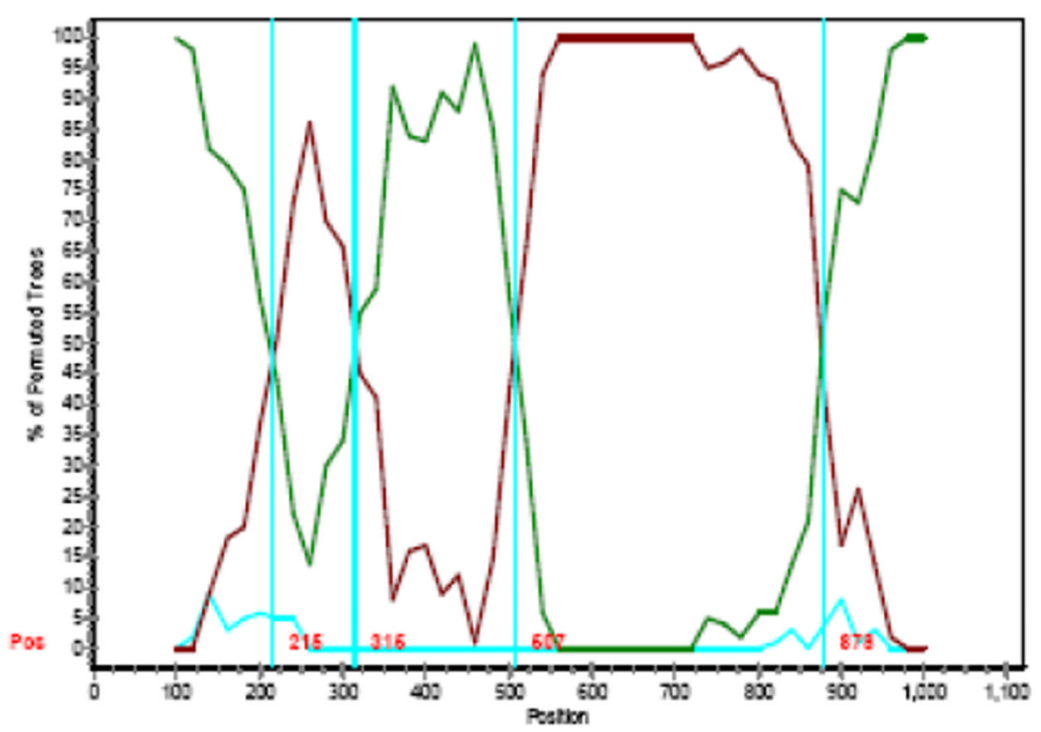




\section{CRF07_BC (97CN001)}

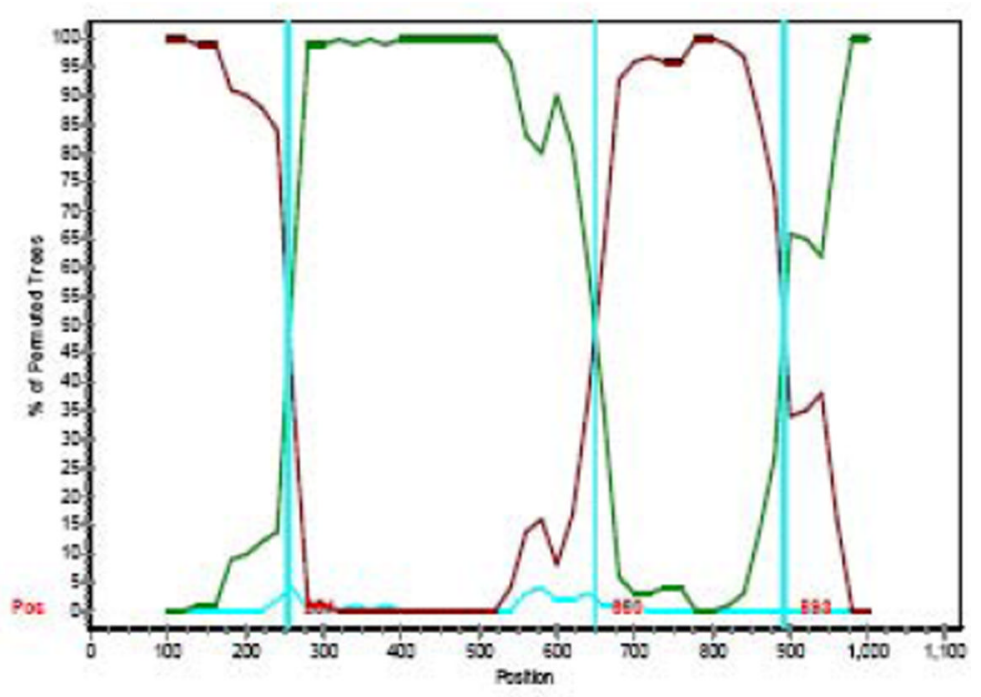

\section{CRF08_BC (98CN006)}

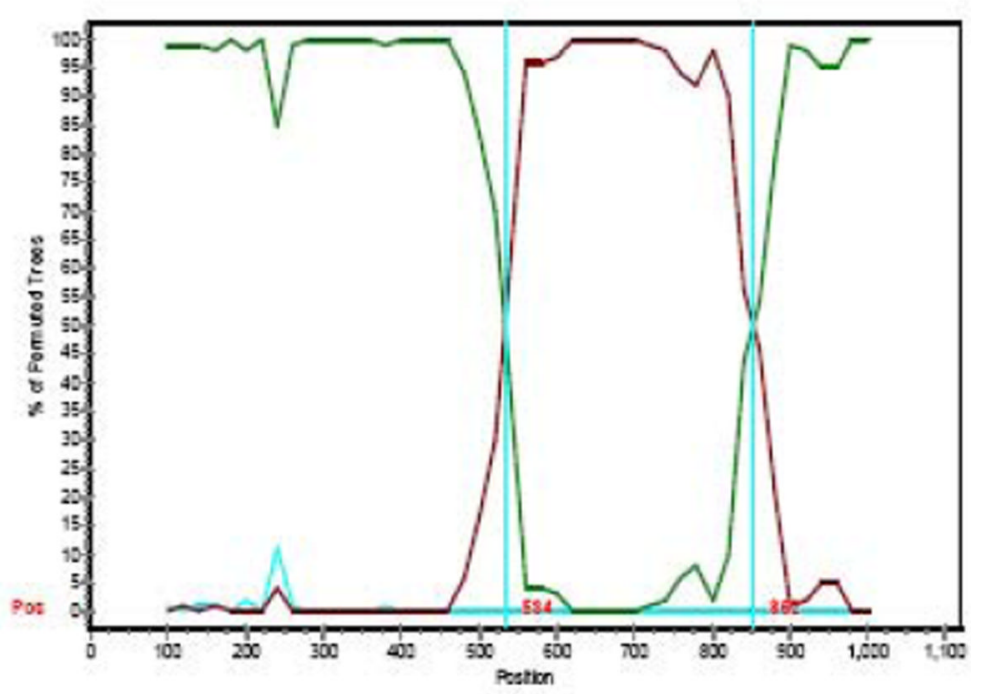

Figure 1.

Bootscanning analyses, to identify new intersubtype recombinant strains. A bootscaning plot was constructed by using Simplot 3.5 .1 software based on 100 replicates with a 200-bp sliding window moving in steps of 20 bases. Subtype references were B' (RL42), India C (95IN21068) and subtype A (KEQ23). The breakpoints in CNYND0019 and CNYND0041 were different from those of the reference strains of CRF07_BC (97CN001) and CRF08_BC (98CN006). 

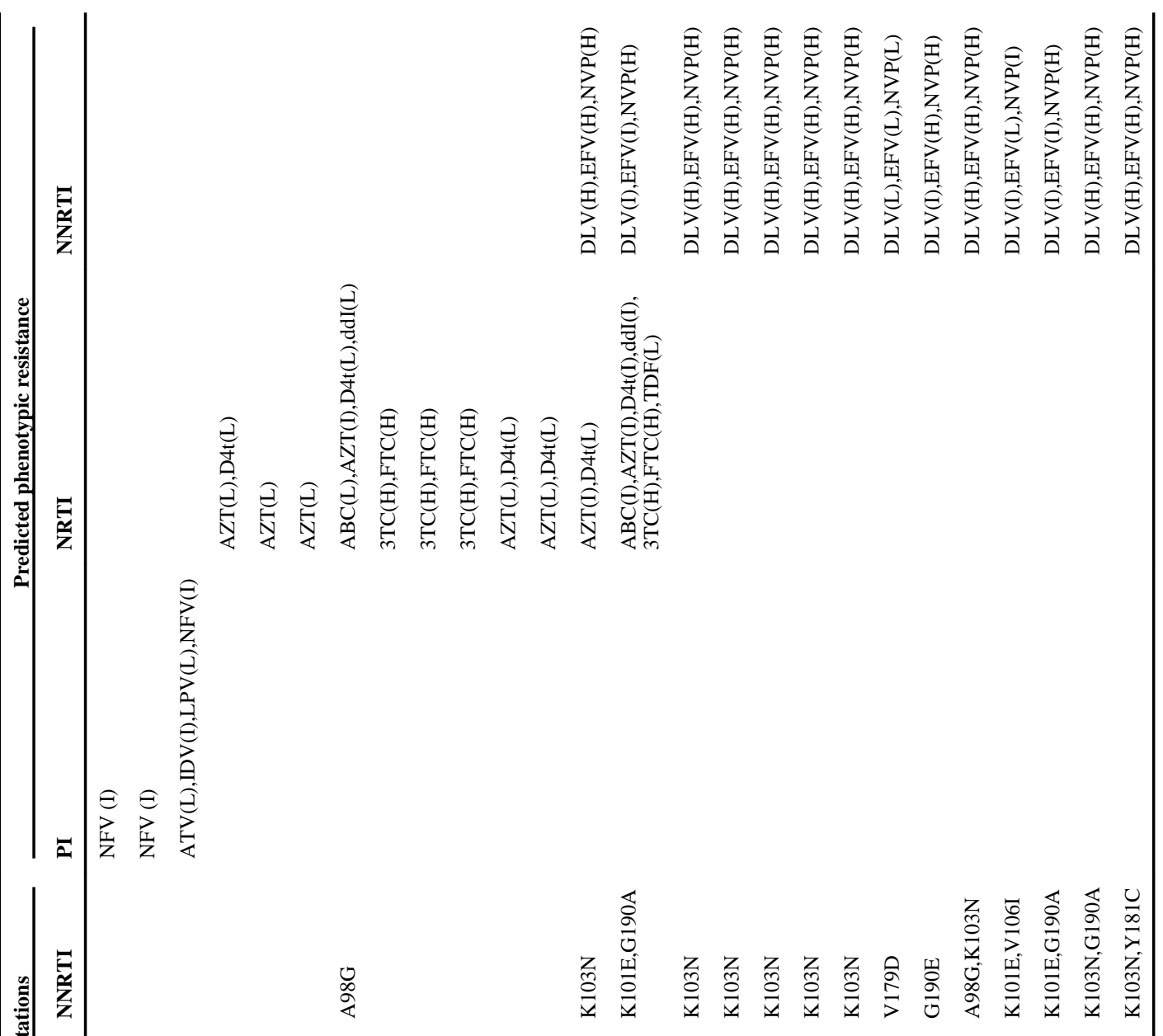

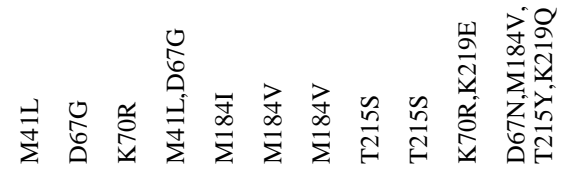

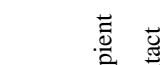


